\title{
Spermiogenesis and Taxonomic Value of Sperm Morphologies of Two Species in Veneridae (Bivalvia: Heterodonta)
}

\author{
Jin Hee Kim ${ }^{1}$ and Sung Han Kim² \\ ${ }^{1}$ Marine Eco-Technology Institute, Busan 608-830, Korea \\ ${ }^{2}$ Department of Aquaculture and Aquatic Sciences, Kunsan National University, Gunsan 573-701, Korea
}

\begin{abstract}
Some characteristics of the formations of acrosomal vesicles during the late stage of spermatids during spermiogenesis and taxonomical charateristics of sperm morphology in male two species (Saxidomus purpurata and Meretrix petechialis) in the family Veneridae were investigated by electron microscope observations. In two species, the morphologies of the spermatozoa have the primitive type and are similar to those of other bivalves in that it contains a short midpiece with five mitochondria surrounding the centrioles. The morphologies of the sperm nuclear types of $S$. purpurata and $M$. petechialis in Veneridae have the curved cylindrical and cylinderical type, respectively. And the acrosome shapes of two species are the same cap-shape type. In particular, the axial filament is not found in the lumen of the acrosome of two species, however, subacrosomal material are observed in the subacrosomal spaces between the anterior nuclear fossa and the acrosomal vesicle of two species. The spermatozoon of $S$. purpurata is approximately $46-52 \mu \mathrm{m}$ in length, including a curved sperm nucleus (about 3.75 $\mu \mathrm{m}$ in length), a long acrosome (about $0.40 \mu \mathrm{m}$ in length),and a tail flagellum (about $45-47 \mu \mathrm{m}$ long). And the spermatozoon of $M$. petechialis is approximately 47-50 $\mu \mathrm{m}$ in length including a slightly curved sperm nucleus (about $1.50 \mu \mathrm{m}$ in length), an acrosome (about $0.56 \mu \mathrm{m}$ in length) and tail flagellum (44-48 $\mu \mathrm{m}$ in length). In two species, the axoneme of the sperm tail flagellum of each species consists of nine pairs of microtubules at the periphery and a pair of cental doublets at the center. Therefore, the axoneme of the sperm tail flagellum shows a 9 +2 structure. In particular, taxonomically important some charateristics of sperm morphologies of two species in the family Veneridae are acrosomal morphology of the sperm, The axial filament is not found in the acrosome as seen in a few species of the family Veneridae in the subclass Heterodonta. The acrosomal vesicle is composed of right, left basal rings and the apex part of the acrosomal vesicle. In particular, right and left basal rings show electron opaque part (region), while the apex part of the acrosomal vesicle shows electron lucent part (region). These charateristics belong to the subclass Heterodonta, unlikely a characteristic of the subclass Pteriomorphia showing all part of the acrosome being composed of electron opaque part (region). Therefore, it is easy to distinguish the families or the subclasses by the acrosomal structures. The number of mitochondria in the midpiece of the sperm of $S$. purpurata and M. petechialis in Veneridae are five. However, the number of mitochondria in the midpiece of the sperm in most species of Veneridae in the subclass Heterodonta are four. Therefore, the number of mitochondria of the sperm midpiece of two species are exceptionally 5 , and it is only exceptional case in the species in Veneridae in the subclass Heterodonta. Except these cases, the number of mitochondria in the sperm midpiece in all families in the subclass Heterodontaare are 4, and now widely used in taxonomic analyses.
\end{abstract}

Key words: Saxidomus purpurata, Meretrix petechialis, spermiogenesis, sperm morphology

\section{INTRODUTION}

Received June 5, 2011 ; Accepted June 26, 2011

Corresponding author: Kim, Jin Hee

Tel: +82 (51) 621-6295 e-mail: jiny90000@hanmail.net

$1225-3480 / 24389$
Spermatogenesis and sperm morphology has been reported in many species of bivalve molluscs using both light and electron microscopy (Eckelbarger et al., 1990; Eckelbarger and Davis, 1996; Gaulejac et al., 
1995; Chung and Ryou, 2000; Chung et al., 2007, 2010; Kim et al., 2010a,b). Recently, in the mollusca, sperm ultrastructure is considered a valuable tool in assessing taxonomic and phylogenetic problems within the bivalvia (Franzén, 1970, 1983; Popham, 1979; Healy, 1989, 1995; Hodgson and Bernard, 1986; . Thus, sperm ultrastructures of bivalves are now widely used in taxonomic analyses (Healy, 1995), and is especially useful when comparing closely related species (Popham et al., 1974; Popham, 1979).

To date, there are several ultrastructural studies on spermatogenesis of the family Veneridae in Korea: Cyclina sinensis (Chung et al., 1991), Meretrix lusoria (Kim, 2006), Gomphina melanaegis (Lee et al., 1999), Gomphina veneriformis (Park et al., 2003; Chung et al., 2010). Protothaca (Notochione) jedoensis (Kim et al., 2010c).

Although there have been several studies on sperm ultrastructure during spermatogenesis of two species (S. purpurata and $M$. petechialis) have been investigated by some authors, there are still gaps in our knowledge on the use of taxonomic characteristics of mature sperm morphology as taxonomic tools to solve the taxonomic problems. Little information is available on the ultrastructural studies associated with spermatogenesis by electron microscopic observation.

Recently, sperm ultrastructures of bivalves are now widely used in taxonomic analyses (Healy, 1995). In particular, it is well-known that acrosomal morphology of sperms has been used to organize bivalve subclasses (Popham, 1979), Therefore, it need to study acrosomal morphology of the sperm and the number of mitochondria in the sperm midpiece for taxonomic analyses of this species. If some characteristics obtained from sperm ultrastructure and the process of spermiogenesis are phylogenetically analyzed, the results of the ultrastructural studies on bivalve spermatozoa will provide information needed for the elucidation of relationship patterns among several bivalve subclasses (Popham et al., 1974, Popham, 1979; Healy, 1989, 1995). Therefore, the main aim of the present study is to describe some taxonomic characteristics for classification from the spermatid and mature sperm morphologies of two species (Saxidomus purpurata and Meretrix petechialis in Veneridae (Heterodonta) by electron microscope observation.

\section{MATERIALS AND METHODS}

\section{Sampling}

For collection of two species (Saxidomus purpurata and Meretrix petechialis), a total of 126 male individuals were collected at the intertidal and subtidal zones of Simpo, Jollabuk-do, Korea, and used for transmission / scanning electron microscope observations.

\section{Transmission electron microscope observations}

For transmission electron microscope observations, excised pieces of the gonads were cut into small pieces and fixed immediately in $2.5 \%$ paraformaldehydeglutaraldehyde in0.1 M phosphate buffer solution $(\mathrm{pH}$ 7.4) for 2 hours at $4{ }^{\circ} \mathrm{C}$. After prefixation, the specimens were washed several times in the buffer solution and then postfixed in a $1 \%$ osmium tetroxide solution in $0.2 \mathrm{M}$ phosphate buffer ( $\mathrm{pH} 7.4$ ) for 1 hour at $4{ }^{\circ} \mathrm{C}$. Specimens then were dehydrated in increasing concentrations of ethanol, cleared in propylene oxide and embedded in an Epon-Araldite mixture. Ultrathin sections of Epon-embedded specimens were cut with glass knives on a Sorvall MT-2 microtome and LKB ultramicrotome at a thickness of about $80-100 \mathrm{~nm}$. Tissue sections were mounted on collodion-coated copper grids, doubly stained with uranyl acetate followed by lead citrate, and observed with a JEM 100 CX-II (80-KV) electron microscope.

\section{Scanning electron microscope observations}

A drop of sperm suspension was placed on a cover glass, prefixed with $2.5 \%$ glutaraldehyde and $2.5 \%$ paraformaldehyde in $0.1 \mathrm{M}$ carcodylate buffer $(\mathrm{pH}$ 7.5 ) at $4^{\circ} \mathrm{C}$ for $15 \mathrm{~min}$, and post fixed with $1 \% \mathrm{OsO}_{4}$ for $10 \mathrm{~min}$, before rinsing with carcodylate buffer. The specimens were dehydrated in a graded ethanol series, critical point dried, coated with gold, and observed under a scanning electron microscope (ISI-SS4D). In addition, after dehydration, some testes were 
freeze-fractured in liquid nitrogen, and then submitted to the same procedure described above.

\section{RESULTS}

\section{Spermiogenesis and sperm morphology of Saxidomus purpurata and Meretrix petechialis}

In general, the process of spermiogenesis of spermatids and mature process of spermatozoa appear to be similar to those of other bivalve species. In this study, to get some taxonomic characteristics for classification from the spermatid and mature sperm morphologies, morphological and ultrastuctural characteristics of spermatids and mature spermatozoa in male Saxidomus purpurata and Meretrix petechialis in Veneridae (Bivalvia: Heterodonta) are investigated by electron microscope observation. Studies on the morphological and ultrastructural characteristics of two species in Veneridae are as follows.

\section{1) Saxidomus purpurata}

Spermatids: For convenience, spermiogenesis has been divided arbitrarily into two stages: the early and late stages. Of them, in the late stage of spermiogenesis, the morphology of the spermatid nucleus changes gradually. At this time, small granules are formed by the Golgi complex in the cytoplasm move to a position just in front of the nucleus, while mitochondria move to a position just behind the nucleus. After all, the morphologies of the spermatid nuclei are gradually elongated, and one or a few granules in the cytoplasm of the spermatid become a proacrosomal vesicle. The proacrosomal vesicle migrate to the presumptive anterior end the spermatid, where they coalesce to form a single electron-dense vesicle. The mitochondria become reduced in number but increase in size by mitochondrial fusion. The larger mitochondria form a close association with the nucleus and in many cases appear tightly apposed to the nuclear envelope. However, the shape of the nucleus is modified and becomes greatly elongated (Fig. 1A).

A proacrosomal vesicle is modified and becomes the cap-shaped acrosomal vesicle on the nucleus. At this time, an acrosomal vesicle is composed of the apex part of the acrosomal vesicle, right and left basal rings, in paticular, right and left basal rings are electron dense opaque parts (region), while the apex part of acrosomal vesicle is electron lucent part (region). In general, these characteristics are one of the ultrastructures of the acrosomal vesicle in the acrosome. At this time, subacrosomal material are present between the anterior part of nuclear fossa and the acrosomal vesicle. However, the axial filaments, which are existed in Ruditapes philippinarum and Gomphina veneriformis in Veneridae, are not found in the subacrosomal material (Fig. 1B). Thereafter, a cap-shaped acrosomal vesicle becomes an acrosome. And then the acrosome lying on the sperm nucleus become a cone in shape. Therefor, the completed acrosome has two regions of differing electron density. It is composed of two long electron-opaque parts (right and left basal rings) and electron-lucent part (apex part). At the same time, the subacrosomal material are present between acrosomal vesicle and the anterior nuclear fossa of the nucleus (Fig. 1C). In the basal part of the nucleus, the mitochondria become reduced in number but increase in size by mitochondrial fusion. Posterior to the nucleus is the midpiece. This region consists of five spherical mitochondria surrounding a pair of triplet substructure centrioles. The cristae of each mitochondrion are randomly arranged. Larger mitochondria form a close association with the nucleus and in many cases appear tightly apposed to the nuclear envelope. At this time, of the two centrioles lying in the midpiece of the spermatozoon, the two centrioles, at right angles, show the classic nine triplets of microtubles (Figs. 1D, E). The proximal centriole laying the posterior fossa of the nucleus lies at $90^{\circ}$ to the sperm longitudinal axis. However, it appears to be unconnected to the nuclear envelope. The distal centriole lies parallel to the sperm longitudinal axis and forms the point of origin for flagellar axoneme (Figs. 1D). During the late spermatid stage, the sperm nucleus is long curved (the angle of the nucleus is about $15^{\circ}$ ), and the cytoplasm is greatly reduced, and so the rate of nucleo-cytoplasm is high. After the sperm nucleus is 

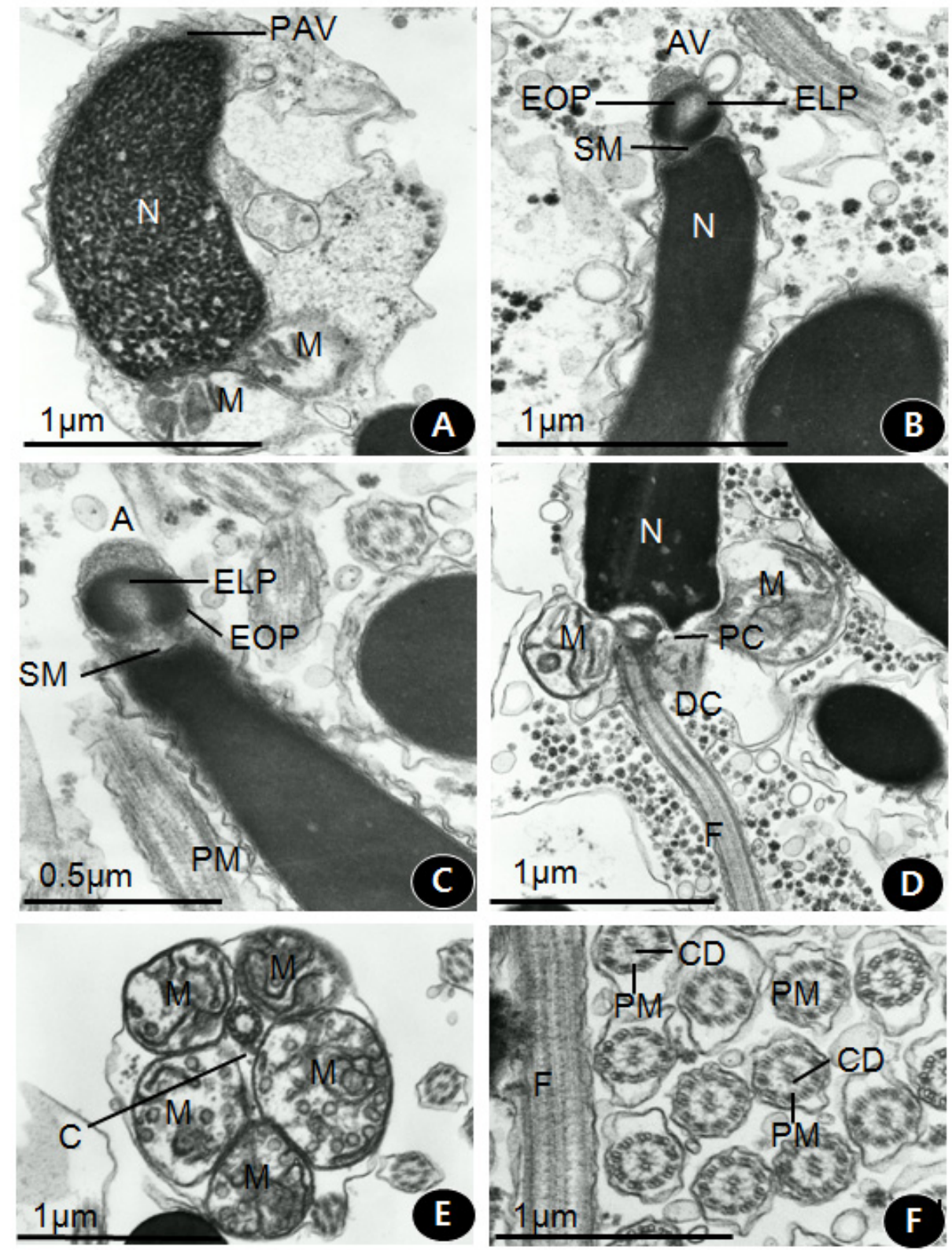

Fig. 1. Transmission electron micrographs of spermiogenesis and spermatozoa in male Saxidomus purpurata (A-F). A, A spermatid (ST) in the late stage of spermatid during spermiogenesis. Note proacrosomal vesicle (PAV) just before the nucleus $(\mathrm{N})$ of the spermatid and the mitochondria (M) near the posterior nuclear fossa; $B$, An acrosomal vesicle (AV) on the elongated, curved spermatid nucleus $(\mathrm{N})$. Note an acrosomal vesicle (AV) being composed of basal rings showing the electron dense opaque part (EOP), electron lucent part (ELP) and subacrosomal material (SM) on the elongated spermatid nucleus; $\mathbf{C}$, An acrosome on a sperm nucleus. Note a modified acrosome consisting of basal rings of the acrosomal vesicle showing electron dense opaque part (EOP), electron lucent part (ELP) and subacrosomal material (SM) on the anterior nuclear fossa of the spermatozoon nucleus surrounded with the plasma membrane; $\mathbf{D}$, Sperm midpiece beneath the curved nucleus $(\mathrm{N})$ and a flagellum. Note the proximal centriole $(\mathrm{PC})$ and distal centriole (DC) with spherical mitochondria (M) beneath the posterior nuclear fossa and a tail flagellum (F); E, Cross sectioned part of the sperm miedpiece. Note five mitochondria surrounding a pair of centrioles; $F$, Cross section of the tail flagella of mature sperm. Note the axoneme showing a 9+2 structure (a pair of central doublets (CD) and nine pair of peripheral microtubules (PM)). 
long elongated, an acrosomal vesicle is also gradually very elongated (average $0.40 \mu \mathrm{m}$ long).

Spermatozoa: After spermiogenesis, differentiation of the spermatozoon is completed. Sperm morphology is the primitive type, as found in most bivalve species that undergo external fertilization. Mature sperm are approximately $46-52 \mu \mathrm{m}$ long. The head is about 4.60 $\mu \mathrm{m}$ long and comprises a long, curved electron-dense nucleus (about $3.75 \mu \mathrm{m}$ ), with a posterior nuclear fossa, and an acrosome about $0.40 \mu \mathrm{m}$ long. The morphology of the sperm nucleus and the acrosomes of this species are of a curved cylindrical type and the cap-shape, respectively (Figs. 1C, D). At this time, a cross- sectioned tail flagellum shows that the axoneme is composed of a $9+2$ substructure (nine peripheral doublets surrounding a central pair of singlet microtubules) enclosed by a plasma membrane (Figs. 1D, F),

\section{Meretrix petechialis.}

Spermatids: In the late stage of spermiogenesis, the morphology of the spermatid nucleus gradually elongated during the differentiation of the spermatid. At this time, one or a few granules, which are formed by the Golgi complex in the cytoplasm of the spermatid, form a proacrosomal vesicle, while mitochondria move to a position just behind the nucleus. The nuclei of spermatids are about $2.9 \mu \mathrm{m}$ long and are cylinderical shape. At this stage, the nucleus shows a granular type. A proacrosomal vesicle migrates to the presumptive anterior end of the spermatid, where they coalesce to form a single electron-dense acrosomal vesicle. A single acrosomal vesicle locates at the presumptive anterior pole of the spermatids, and the subacrosomal material appear between the acrosomal vesicle and the anterior nuclear fossa of the nucleus (Fig. 2A). The acrosomal vesicle is initially oval in shape, but gradually assumes a cap-like form with invaginated posterior face. Two components of the acrosomal vesicle can be recognized: the acrosomal vesicle and an extensive deposit of subacrosomal (extravesicular) material. The acrosomal vesicle is membrane bound, become cap-shaped form by way of various morphorphological changes, and its size is about $0.56 \mu \mathrm{m}$ long. Contents of the acrosomal vesicle are finely granular, moderately electron dense. At this stage, after mitochondria move to a position just behind the nucleus, several small mitochondria are fused each other and become larger ones near the posterior nuclear fossa of the nucleus. The morphologies of many cristae of mitochondria are irregular (Fig. 2B).

As the late stage of spermatid develops gradually, granular nuclei of spermatids change into fibrous ones, and then rapidly they change to narrow and slightly elongated curved nucleus (the angle of the nucleus is $10^{\circ}$ ). A curved acrosomal vesicle is composed of right, left basal rings and subacrosomal materials in the space between the anterior nuclear fossa and the basal rings. At this time, in particular, right and left basal rings are electron dense opaque part (region), while the apex part of acrosomal vesicle is electron lucent part (region). Subacrosomal material are electron moderate part.

In the basal part of the nucleus, the mitochondria become reduced in number but increase in size by mitochondrial fusion. Posterior to the nucleus is the midpiece. This region consists of five spherical mitochondria surrounding a pair of triplet substructure centrioles. The cristae of each mitochondrion are randomly arranged. Larger mitochondria appear tightly apposed to the nuclear envelope (Fig. 2C). At this time, of the two centrioles lying in the midpiece of the spermatozoon, the two centrioles, at right angles, show the classic nine triplets of microtubles. The proximal centriole laying the posterior fossa of the nucleus lies at $90^{\circ}$ to the sperm longitudinal axis. However, it appears to be unconnected to the nuclear envelope. The distal centriole lies parallel to the sperm longitudinal axis and forms the point of origin for flagellar axoneme . During the late spermatid stage, the sperm nucleus is slightly curved (the angle of the nucleus is about $10^{\circ}$ ), and the cytoplasm is greatly reduced, therefore, the rate of nucleo-cytoplasm is high. After the sperm nucleus is elongated, an acrosomal vesicle is also gradually elongated (about $0.56 \mu \mathrm{m}$ long, Fig. 2D). 


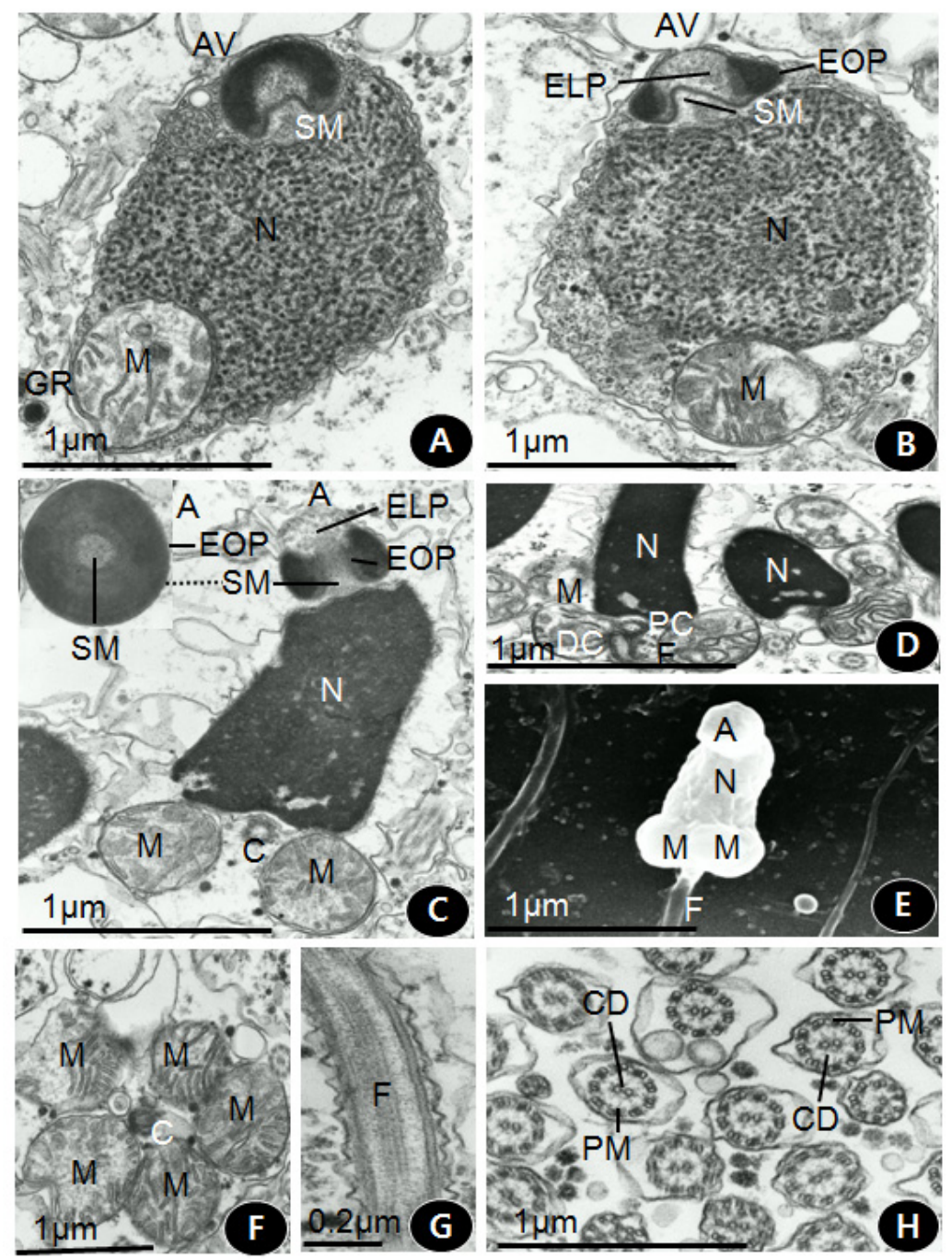

Fig. 2. Transmission / scanning electron micrographs of spermiogenesis and spermatozoa in male Meretrix petechialis (A-H). A, A spermatid (ST) in the late stage of spermatid during spermiogenesis. Note granules $(\mathrm{GR})$, acrosomal vesicle (AV) and subacrosomal material just before the spermatid nucleus (N) of the spermatid and the mitochondria (M) near the posterior nuclear fossa; B, An acrosomal vesicle (AV) on granular spermatid nucleus $(N)$. Note an acrosomal vesicle (AV) being composed of basal rings showing the electron dense opaque part (EOP), electron lucent part (ELP) and subacrosomal material (SM) on the spermatid nucleus; C, An acrosome on a sperm nucleus and the midpiece of the sperm. Note a modified acrosome consisting of the basal rings of acrosomal vesicle showing electron dense opaque part (EOP), electron lucent part (ELP) and subacrosomal material (SM) on the anterior nuclear fossa of the sperm nucleus $(\mathrm{N})$ and the centrioles and large mitochondria $(\mathrm{M})$ in the midpiece of the sperm. Cross sectioned an acrosome being compose of acrosomal vesicle showing e lectron dense opaque parts and subacrosomal material (SM); D, Sperm midpiece and a flagellum beneath the nucleus $(\mathrm{N})$. Note the proximal centriole $(P C)$ and distal centriole (DC) with spherical mitochondria $(M)$ beneath the posterior nuclear fossa and a tail flagellum (F); E, Scanning electron micrograph. Longitudinal sectioned a sperm. A completed spermatozoon is composed of the head part including acrosome $(A)$ and nucleus $(N)$, the midpiece part, and the tail flagellum part $(F) ; F$, Cross sectioned part of the sperm midpiece. Note five mitochondria surrounding a pair of centrioles; G, H, Longitudinal section $(G)$ and cross section $(H)$ of the tail flagella of mature sperm. Note the axoneme showing a $9+2$ structure (a pair of central doublets $(C D)$ and nine pair of peripheral microtubules (PM)). 
Spermatozoa: In the mature stage, the morphology of the spermatozoon has a primitive type and is similar to those of other bivalves. The spermatozoon of M. petechialis is approximately 47-50 $\mu \mathrm{m}$ in length including a slightly curved sperm nucleus $(\mathrm{N})$ (about $1.50 \mu \mathrm{m}$ in length), an acrosome (A) (about $0.56 \mu \mathrm{m}$ in length) and tail flagellum (44-48 $\mu \mathrm{m}$ in length). At this time, several lacunae are found in the nucleus (Fig. E).

At this time, in particular, an acrosome on th nucleus is composed of the acrosomal vesicle (which comprises both right and left basal rings) and subacrosomal material (Figs. 2C, D). In the midpiece of the spermatozoon, this region consists of five equal-sized, spherical mitochondria surrounding a pair of triplet substructure centrioles (Fig. 2F). The cristae of each mitochondrion are randomly arranged., The initial portion of the flagellum attached to the plasma membrane (Fig. 2G). Transverse sections reveal that the triplets of the distal centriole posteriorly transform into doublet are continuos with the nine doublets of the flagellar axoneme. The flagellum is composed of a $9+2$ substructure axoneme (that is, nine peripheral microtubules surrounding a pair of central doublets enclosed by the plasma membrane (Figs. 2G, H).

\section{DISCUSSION}

In the present study, morphology and the ultrastructure of spermatozoa during spermatogenesis in two species (S. purpuratus and M. petechialis) are similar to those of ther bivalves, In particular, spermiogenesis of two species also shows similar phenomena to those of other bivalves (Hodgson and Bernard, 1986; Eckelbargeret al., 1990; Healy and Lester, 1991; Chung et al., 1991; Eckelbarger and Davis, 1996; Chung and Ryou, 2000; Park et al., 2006; Chung et al., 2007; Kim et al., 2010 a,b,c,d)

Regarding the morphologies of the sperm nuclei in Veneridae species, Kim (2001) reported that the angles of the sperm nuclei in the family Veneridae ranged from $0^{\circ}$ (Notochione jedoensis) to $80^{\circ}$ (Mercenaria stinpsoni). In this study, the angles of the sperm nuclei of $S$. purpurata was slightly curved $\left(15^{\circ}\right)$, however, exceptionally, that of this species was smaller than those of Dosinorbis japonicus $\left(45^{\circ}\right)$ and G. melanaegis $\left(9.54^{\circ}\right)$. Thus, the morphologies of sperm nuclei vary with the species. Therefore, morphologies of the sperm nuclei can not be used for classifications of the species, genuses, families and subclasses as taxonomic tool.

Regarding the morphology of sperm acrosome, $S$. purpuratus and M. petechialis are the cap shape (Kim, 2001). Compare two species with the morphology of the acrosomal vesicles in species of other families, the morphological, phylogenetical characteristics of acrosomal vesicles in S. purpurata and M. petechialis are the cap shape during spermatogenesis. Therefore, we assume that the presence of a special acrosomal vesicle during spermatogenesis can be used as a key characteristic for identification of species of two genuses Saxidomus and Meretrix, as seen in the family Veneridae.

\section{Taxonomic value of sperm morphology and ultrastructure}

Recently, sperm ultrastructure of bivalves is considered a valuable tool in assessing taxonomic and phylogenetic problems within the Bivalvia (Franzén, 1970, 1983; Popham, 1979; Eckelbarger et al., 1990), and it is now widely used in taxonomic analyses (Healy, 1995): for example, 1) acrosomal morphology, and 2) the number of mitochondria in the sperm midpiece.

From the results investigated, we have confirmed that the acrosomes can be distinguisable those of the genuses, families and subclasses (Pteriormorphia and Heterodonta) by the morphologies, positions and characteristics of the acrosomal vesicle. Family Veneridae belongs to the subclass Heterodonta.

According to Hodgson and Bernard (1986), in general, all species in the subclass Heterodonta in the bivalves have a common structural characteristics of the acrosomal vesicles showing the cap-like or cone-like in shape, being composed of electron high density (opaque) materials (right and left basal rings) and electron lucent materials (the apex part of the acrosomal vesicle). 
In addition, Hodgson and Bernard, 1986 reported that the subclass Pteriomorphia in the bivalves have a common structural characteristics of the acrosomal vesicles showing the cone-like in shape, being composed of the electron high density (opaque) part in all parts.

In this study, two species ( $S$. purpurata and $M$. petechialis) that belong to the family Veneridae in the subclass Heterodonta, have the acrosomal vesicles showing the cap in shape, being composed of electron high density (opaque) materials (right and left basal rings) and electron lucent materials (the apex part of acrosomal vesicle) as reported by Hodgson and Bernard (1986). Therefore, S. purpurata and $M$. petechialis in Veneridae belong to the subclass Heterodonta because our results coincided with the opinion of Hodgson and Bernard (1986).

Regarding the number of mitochondria in the midpiece of the sperms, Kim (2001) reported that the number of mitochondria in the midpiece of the sperms of the species in the family Veneridae are four in $\mathrm{n}$ most specie, while exceptionally, five in $S$. purpurata, M. lusoria and C. sinensis. Exceptionally, within one species, the number of mitochondria in the midpiece of the sperm show slight differences in number. Regarding the number of mitochondria in the sperm midpiece, Healy (1995) reported that the number of mitochondria tends to be stable within any given family or superfamily. Therefore, we agree with the opinion of Healy (1995).

\section{ACKNOWLEDGEMENTS}

The authors are grateful to Professor Emeritus Ee-Yung Chung, Kunsan National University, for helpful comments on the manuscript.

\section{REFERENCES}

Chung, E.Y., Lee, T.Y. and An, C.M. (1991) Sexual maturaration of the venus clam, Cyclina sinensis, on the west coast of Korea. Journal of Medical and Applied Malacology, 3: 125-136.

Chung, E.Y. and Ryou, D.K. (2000) Gametogenesis and sexual maturation of the surf clam Mactra venerifermis on the west coast of Korea. Malacologia, 42: 149-163.

Chung, E.Y., Kim, E.J. and Park, G.M. (2007)
Spermatogenesis and sexual maturation in male Mactra chinensis (Bivalvia: Mactridae) of Korea. Integrative Bioscience, 11: 227-234.

Chung, E.Y., Chung, C.H., Kim, J.H., Park, S.W. and Park, K.H. (2010) Ultrastructures of germ cells and the accessory cells during spermatogenesis in male Gomphina veneriformis (Bivalvia: Veneridae) on the East Sea of Korea. Korean Journal of Malacology, 26: $51-62$.

Eckelbarger, K.J., Bieler, R., and Mikkelsen, P.M. (1990) ltrastructure of sperm development and mature sperm morphology in three species of commensal bivalves (Mollusca: Galeommatoidea). Journal of Morphology, 205: 63-75.

Eckelbarger, K.J. and Davis, C.V. (1996) Ultrastructure of the gonad and gametogenesis in the eastern oyster, Crassostrea virginica. II. Testis and spermatogenesis. Marine Biology, 127: 89-96.

Franzén, A. (1970) Phylogenetic aspects of the mophology spermatozoa and spermiogenesis. In; Baccetti B (ed) "Comparative spermatology.". Accademia Nationale Dei Lincei, Rome, pp. 573.

Franzén, A. (1983) Ultrastructural studies of spermatozoa in three bivalve species with notes on evolution of elongated sperm nucleus in primitive spermatozoa. Gamete Research, 7: 199-214.

Gaulejac, de J., Jenry, M. and Vicente, N. (1995) An ultrastructural study of gametogenesis of the marine bivalve Pinna nobilis (Linnaeus, 1758). II. Spermatogenesis. Journal of Molluscan Studies, 61: 393-403.

Healy, J.M. (1989) Spermiogenesis and spermatozoa in the relict bivalve genus Neotrigonia: relevance to trigonioid relationships, particularly Unionoidea. Marine Biology, 103: 75-85.

Healy, J.M. (1995) Sperm ultrastructure in the marine bivalve families Carditidae and Crassatellidae and and its bearing on unification of the Crasssatelloidea with the Carditoidea. Zoological Science, 24: 1-28

Healy, J.M. and Lester, R.J.G. (1991) Sperm ultrastructure in the Australian oyster Saccostrea commercialis (Iredale and Roughley) (Bivalvia: Ostreidea), Journal of Molluscan Studies. 57: 219-224.

Hodgson, A.N. and Bernard, R.T.F. (1986) Ultrastructure of the sperm and spermatogenesis of three species of Mytilidae (Mollusca, Bivalvia). Gamete Research, 15: $123-135$.

Kim, J.H. (2001) Spermatogenesis and comparative ultrastructure of spermatozoa in several species of Korean economic bivalves (13 families, 34 species). Pukyung National University 161 pp.

Kim, J.H., Chung, E.Y., Choi, K.H., Park, K.H. and Park, S.W. (2010a) Ultrastructure of germ cells during spermatogenesis and some characteristics of sperm morphology in Male Mytilus coruscus (Bivalvia: Mytilidae) on the west coast of Korea. Korean Journal of Malacology, 26: 33-43. 
Kim, J.H., Chung, E.Y., Choi, K.H., Lee, K.Y. and Choi, M.S. (2010b) Ultrastructure of the testis and germ cell development during spermatogenesis in male Crassostrea gigas (Bivalvia: Ostreidae) in western Korea. Korean Journal of Malacology, 26: 235-244.

Kim, J.H., Park, Y.J., Lee, K.Y., Choi, M.S., Seo, W.J. and Chung, E.Y. (2010c) Germ cell differentiations during spermatogenesis and ultrastructural characteristics of mature sperms in male Protothaca (Notochione) jedoensis (Bivalvia: Veneridae). Development and Reproduction, 14: 269-279.

Kim, J.H., Chung, E.Y., Lee, K.Y., Choi, M.S. Seo, W.J. and Kim. S.H. (2010d) Spermatid Differentiations during spermiogenesis and mature sperm ultrastructure in male Crassostrea niponica (Seki, 1934) (Pteriomorphia: Ostreidae). The Korean Journal of Malacology, 26: 311-316.

Popham, J.D. (1974) Comparative morphometrics of the acrosomes of the sperms of externally and internally fertilizing sperms of the sperms of the shipworms (Teredinidae, Bivalvia, Mollusca). Cell Tissue Research. 150: 291-297.

Popham, J.D. (1979) Comparative spermatozoon morphology and bivalve phylogeny. Malacological Review, 12: 1-20. 